\title{
RECOVERY AND COUNTER-CURRENT SEPARATION OF INDIGO AND INDIRUBIN FROM POLYGONUM TINCTORIUM AIT. LEAVES
}

\section{(C) Kukula-Koch Wirginia, Glowniak Kazimierz}

Chair and Department of Pharmacognosy with Medicinal Plant Unit, Medical University of Lublin, 1, Chodzki str., 20093 Lublin, Poland, virginia.kukula@gmail.com

Indigo naturalis ("Qing dai") has been used in traditional Chinese medicine (TCM) as a heat remover. Polygonum tinctorium Ait. belongs to the indigoferous plants together with Indigofera tinctoria L., Isatis indigotica Fort., Isatis tinctoria L., and Baphicacanthus cusia Brem. Its leaves are rich sources of indigo dye, indirubin (a well-known antipsoriatic and antibacterial agent), isatan $B$, and tryptanthrine. Interest in the drug was fostered when activity against myelocitic leukemia of indirubine was discovered. Currently indirubin is known to inhibit cyclin-dependent kinases (CDK1/cyclin B and CDK5/p25) as well. Current report is to show the recent advances in the isolation of the precious constituents of $P$. tinctorium leaf extracts. Suggested by Chinese Pharmacopoeia extraction protocols remain unfavourable, as they employ the usage of highly toxic solvents (chloro- form). Herewith we present the optimization of extraction process, introducing the $2 \%$ solution of chloral hydrate in dichloromethane as the best from the investigated solvent systems characterized by lower toxicity. Separation of $P$. tinctorium Ait. metabolites was performed using environmentally friendly separatory technique: Fast Centrifugal Partition Chromatography. Among several tested solvent systems, the mixture of $n$-heptane: ethyl acetate: methanol: water (6:1:6:1) was perceived as the best one. The one led to obtaining of indigo and indirubin of high purity already after the first injection of crude extract. Indigo content in $P$. tinctorium Ait. samples from Taiwan were found to be similar to its quantity determined in the leaves grown in Poland and collected in the late summer. It may prove, that the cultivation of the investigated species could be spread to Europe.

\section{A STUDY ON PERIPHERAL UTILIZATION OF GLUCOSE AND ALDOSE REDUCTASE INHIBITORY ACTIVITY OF HOUTTUYNIA CORDATA FRACTIONS}

\section{(C) Kumar Manish, Laloo Damiki, Prasad Satyendra K., Hemalatha S}

Department of Pharmaceutics, Institute of Technology, Banaras Hindu University, Varanasi-221005, India, Email: mkumar. rs.phe@itbhu.ac.in

The plant Hottuynia cordata, used as a medicinal salad for lowering the blood sugar level in the North Eastern parts of India. Our earlier investigation showed significant antidiabetic effect of $H$.cordata in streptozotocin induced diabetic rats (1). Therefore, the aim of present study was to evaluate the glucose uptake by isolated rat hemi-diaphragm in an in vitro model. We have also evaluated in vitro aldose reductase inhibitory activity (ARI) and their kinetic profile on different fractions of $H$. cordata Thunb. (Saururaceae) for their potential use in diabetic cataract. Total phenolic and flavonoids content of different fraction of H.cordata was determined by Folin-Ciocalteu and aluminium chloride method respectively. Glucose uptake by isolated rat hemi-diaphragm was evaluated in different fractions of $H$. cordata using insulin as a reference standard. Rats lens were enucleated through posterior approach and their homogenate was prepared and centrifuged to obtain clear supernatant for the determination of AR activity and protein content. The kinetic studies of inhibitory activity against aldose reductase of different fractions were analyzed using the Lineweaver-Burk plot with varying concentrations of glyceraldehydes. $\mathrm{K}_{\mathrm{m}}$ and $\mathrm{V}_{\max }$ were also estimated. $\mathrm{K}_{\mathrm{i}}$ value in presence of different fractions was determined by applying Cheng-Prusoff equation (2). The result showed significant level of phenol and flavonoids in ethyl acetate and aqueous fraction compared to hexane and chloroform fraction. Ethyl acetate fraction showed maximum glucose utilization by rat hemidiaphragm and was also found to have the most potent ARI activity which was significant when compared with all other fractions. Their $I_{50}$ values with descending order of activity was reported to be: ethyl acetate [64.62 $\pm 3.9 \mu \mathrm{g} / \mathrm{ml}]>$ aqueous $[90.69 \pm 7.5 \mu \mathrm{g} / \mathrm{ml}]>$ hexane $[134.59 \pm 4.9 \mu \mathrm{g} / \mathrm{ml}]>$ chloroform $[151.58 \pm 3.3 \mu \mathrm{g} / \mathrm{ml}]$ respectively. From the kinetic studies, it was found that the ethyl acetate fraction showed a non-competitive inhibition while all other fractions showed competitive inhibition. The present study concluded that ethyl acetate fraction of H.cordata exhibited maximum glucose utilization and ARI activity which may be due to the presence of high phenols and flavonoids content.

References: (1) Kumar M, Prasad SK, Laloo D, Hemalatha S. 2011. $12^{\text {th }}$ congress of International Society for Ethnopharmacology, Kolkata India, Feburary 17-19, Abstract No. SNPSJU0279. (2) Daniellou R, Zheng H, Palmer DRJ. 2006. Can $J$ Chem 84: 522-527. 